\title{
Perfil microbiológico em farinha de milho flocada
}

\author{
Microbiological profile in flaked corn flour \\ Perfil microbiologico em harina de maíz flocada
}

Neide Kazue Sakugawa Shinohara

ORCID: https://orcid.org/0000-0001-8356-874X Universidade Federal Rural de Pernambuco, Brasil E-mail: neideshinohara@gmail.com

Allan Matheus de Souza Silva

ORCID: https://orcid.org/0000-0003-1096-7445 Universidade Federal Rural de Pernambuco, Brasil E-mail: Allanmatheus10@ hotmail.com

Felipe Araújo de Souza Leão

ORCID: https://orcid.org/0000-0002-9533-2475 Universidade Federal Rural de Pernambuco, Brasil

E-mail: felipeleao.nutri@gmail.com

Thiago Ramos da Silva

ORCID: https://orcid.org/0000-0002-0453-8794 Universidade Federal Rural de Pernambuco, Brasil E-mail: thiagorammos@gmail.com

Amanda Mirelly Santos Sobral

ORCID: https://orcid.org/0000-0002-6598-1140 Universidade Federal Rural de Pernambuco, Brasil E-mail: amandamssobral@gmail.com

Patrícia Karla Batista de Andrade ORCID: https://orcid.org/0000-0002-3036-5719 Universidade Federal Rural de Pernambuco, Brasil E-mail: patriciapig2@yahoo.com.br Jucarlos Rufino de Freitas

ORCID: https://orcid.org/0000-0002-3497-4263 Universidade Federal Rural de Pernambuco, Brasil E-mail: jucarlos123@hotmail.com

Moacyr Cunha Filho

ORCID: https://orcid.org/0000-0002-3466-8143 Universidade Federal Rural de Pernambuco, Brasil E-mail: moacyr.cunhafo@ufrpe.br

\section{Resumo}

A farinha de milho flocada (FMF) está presente no café matinal do nordestino, demarcando a forte presença do milho na rotina dietética. O objetivo foi avaliar a qualidade sanitária da FMF comercializada na Região da Grande Recife (RGR), localizado no nordeste do Brasil. Foram realizados ensaios biológicos para investigação de microrganismos patogênicos e deteriorantes. Não foram detectados a presença de enteropatógenos previstos na legislação sanitária brasileira, entretanto, foi isolado microbiota acompanhante, como leveduras mesofílica e bolores termorresistente. Apesar da detecção desses agentes biológicos, a concentração se encontra em conformidade com a recomendação sanitária. Entretanto, a presença destes fungos nos alimentos, podem produzir metabólitos secundários (micotoxinas), cuja presença podem evoluir para quadros carcinogênicos e hepatotóxico, principalmente em grupos vulneráveis, provocando assim, grande desordem na saúde pública.

Palavras-chave: Zea mays; Cereal; Contaminação fúngica.

\section{Abstract}

Flaked corn flour (FCF) is very present at the breakfeast, demarcating the Strong presence of corn on northeastern diet. The objective was evaluate the sanitary quality of the FCF marketed on Região da Grande Recife (RGR), trhough biological assays to investigation of petogenic microrganisms provided for in legislation of patogenics and deteriorating microsganisms. Were not detected the presence of enteropatogenic provided for in sanitary legislation, however, accompainyng microbiota was detected, such as mesophiles mold, yeast and termo-resistant fungi. Although the detection on those biological agentes, the concentration was conforming with the sanitary recomendation. However, the presence of microrganisms able to pruduce secondary metabolits, such as mycotoxins, may evolve into 
carcinogenic and hepatotoxic conditions mainly in vulnerable groups, what may provoke a great disorder at public health.

Keywords: Zea mays; Cereal; Fungal contamination.

\section{Resumen}

La harina de maíz flocada (FMF) está presente en el café matutino del Nordeste, demarcando la fuerte presencia del maíz en la rutina dietética. El objetivo fue evaluar la calidad sanitaria de la FMF comercializada en la Región del Gran Recife (RGR), ubicada en el noreste de Brasil. Se realizaron pruebas biológicas para investigar microorganismos patógenos y en deterioro. No se detectó la presencia de enteropatógenos previstos en la legislación sanitaria brasileña, sin embargo, se aisló microbiota acompañante, como levaduras mesófilas y mohos termorresistentes. A pesar de la detección de estos agentes biológicos, la concentración cumple con la recomendación sanitaria. Sin embargo, la presencia de estos hongos en los alimentos, puede producir metabolitos secundarios (micotoxinas), cuya presencia puede evolucionar a condiciones cancerígenas y hepatotóxicas, especialmente en grupos vulnerables, provocando así un gran trastorno de salud pública.

Palabras clave: Zea mays; Cereal; Contaminación por hongos.

\section{Introdução}

O milho é nativo das civilizações asteca, maia e inca, onde há séculos o homem consome na rotina alimentar (Cascudo, 1998; Santos, Mendonça, Shinohara, 2020). O milho (Zea mays) é um grão que pode ser consumido fresco, seco ou seus derivados como a farinha de milho, fubá, quirela, canjiquinha e farinha flocada (Araújo et al., 2016; KOVESI et al., 2007). No caso da farinha de milho flocada ou flocos de milho pré-cozidos, é um produto obtido por meio da laminação de diferentes frações dos grãos de milho germinado (Brasil, 2017).

É um cereal muito versátil e bastante utilizado na alimentação humana e animal, considerado alimento energético devido ao alto percentual de carboidratos, e muito consumido no território nacional na forma de farinha de milho (Amaral et al., 2019). No Brasil e no mundo, a produção de grãos é um dos principais setores do segmento agrícola, tendo o milho, grande participação no mercado mundial (Tiecker et al., 2014; Simão et al., 2020; Ventura et al., 2020). A estimativa nacional de produção de milho, considerando a primeira, segunda e terceira safras, na temporada 2019/20, está estimada em 101,9 milhões de toneladas, volume semelhante ao da safra 2018/19, que mesmo com o cenário pandêmico da COVID-19 enfrentada pelo mundo, não afetará o bom andamento da safra brasileira e na pauta da exportação em 2020 (CONAB, 2020).

A contaminação fúngica e a infestação por insetos, são as principais causas da deterioração do milho, os fungos podem causar de 50 a $80 \%$ de perdas nos grãos durante o armazenamento em silos, se as condições de temperatura, umidade relativa e controle integrado de pragas não estiverem controladas para evitar a multiplicação biológica e a infestação de pragas. Os principais fungos associados ao armazenamento dos cereais, incluem espécies dos gêneros Aspergillus e Penicillium, associado a ausência das Boas Práticas Agrícolas (Negasa, Solomon, Girma, 2019).

Os alimentos para consumo humano estão sujeitos a contaminação e deterioração por fungos, podendo levar a produção de toxinas como metabólitos secundários, que são compostos químicos que representam sério risco à saúde humana e de animais (Onyedum et al., 2020). Dependendo da concentração celular ou exposição frequente aos agentes fúngicos, estes podem levar a formação de metabólitos tóxicos nos alimentos e quando consumido pelos humanos, eleva o risco de causar efeitos crônicos, como o aparecimento de câncer nos principais órgãos, comprometimento do sistema imunológico e hepático, e retardo do crescimento inflantil (Ayeni et al., 2020).

Os derivados do milho são muito utilizados e bastante apreciados na culinária brasileira. A farinha de milho flocada (FMF) é o ingrediente principal do cuscuz matinal do nordestino, onde a farinha é temperada com sal e cozida no vapor. Essa preparação adquire consistência de farofa úmida, podendo ser servida com leite, leite de coco, abóbora, ovo ou queijo coalho (Araújo et al., 2016; Freixa, Chaves, 2012; Santos, Furtado, Shinohara, 2019). Diante do consumo do milho na rotina alimentar 
no nordeste brasileiro, a pesquisa objetivou avaliar a qualidade sanitária da farinha de milho flocada e comercializada na Região da Grande Recife (RGR), em Pernambuco.

\section{Metodologia}

Foram adquiridas 31 (trinta e uma) amostras cujas embalagens estavam íntegras com 500 gramas de peso líquido, de diferentes marcas de farinha de milho flocada, comercializadas em 6 (seis) municípios localizados na Região da Grande Recife (Figura 1), no estado de Pernambuco, Brasil. Todas as amostras analisadas se apresentavam dentro do prazo de validade, para garantia da avaliação sanitária. As análises foram realizadas no Laboratório de Alimentos e Ambiental, do Departamento de Tecnologia Rural, na Universidade Federal Rural de Pernambuco.

Figura 1: Localização geográfica dos municípios em estudo das FMF na Região da Grande Recife (RGR) em PE, nordeste do Brasil.

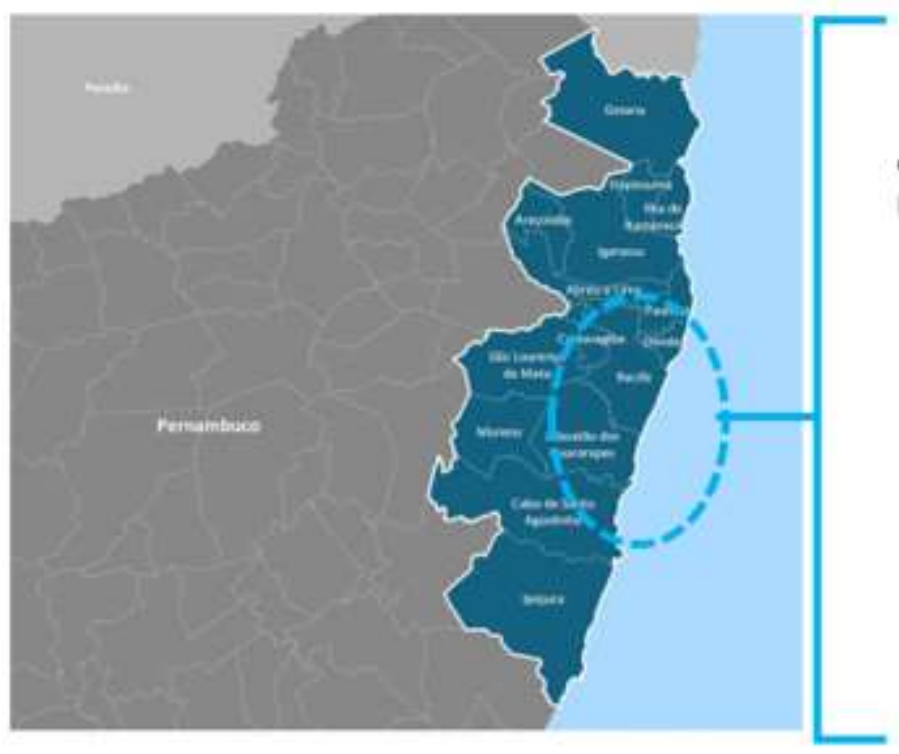

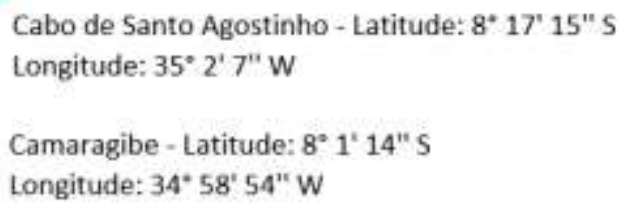

São Lourenço da Mata - Latitude: 8" 0' $13^{\prime \prime}$ S Longitude: $35^{\circ} 1^{\prime} 17^{\prime \prime} \mathrm{W}$

Fonte: Google Maps (2020).

As análises microbiológicas empregaram kits comerciais para a contagem total de aeróbios mesófilo (Compact Dry TC®), Escherichia coli (Compact Dry EC®), e detecção da Salmonella spp. (Compact Dry SL®). A investigação na contagem de Coliforme total, Bacillus cereus, Bolores e Leveduras e Bolores Termorresistentes, seguiram marcha analítica de Silva e colaboradores (2017), APHA (2005) e ICMSF (2002).

As metodologias e parâmetros microbiológicos atenderam as normas sanitárias RDC 331 (Brasila, 2019) e IN 60 (Brasilb, 2019). Ambas as legislações em vigor não determinam obrigatoriedade para contagem total de aeróbios mesófilo, Bolores, Leveduras e Bolores Termorresistentes, parâmetros microbiológicos que foram adicionados a essa pesquisa, para promover maior abrangência e segurança na investigação da qualidade sanitária das FMF e que são comercializadas na RGR.

As metodologias empregadas são aprovadas pela Codex Alimentarius, APHA, e AOAC para ensaios microbiológicos de alimentos. Todos os ensaios biológicos foram realizados em duplicata e os resultados estão expressos em Unidades Formadoras de Colônias por grama (UFC/g) e ausência ou presença para Salmonella spp.

Para a análise estatística das variáveis microbiológicas foi aplicado análise descritiva, através do teste de ShapiroWilk e expressa em gráfico, sendo considerada a normalidade estatisticamente significativa valores que apresentaram p-valor superior a 5\% (Shapiro, Wilk, 1965). 


\section{Resultados e Discussão}

Segundo Franco (2012) e Germano e Germano (2019), a inspeção sanitária tem como missão investigar e identificar as prováveis fontes, vias e agentes de contaminação nos alimentos, para assim dimensionar os efeitos deletérios nos processos tecnológicos da produção. Massaguer (2005) e Silva et al. (2017) citam que nos casos de surtos de origem biológica, principalmente na detecção dos agentes esporulados, quando presentes no alimento, representam grande severidade sanitária, pois possuem a capacidade de sobrevivência e proliferação em altas temperaturas, assim metodologias oficiais para identificação de microrganismos termófilos se fazem necessário, para mitigar o risco de surtos alimentares não previstos, junto aos consumidores.

Nessa pesquisa, inicialmente foi realizado a conversão de Unidade Formadora de Colônia por grama (UFC/g) para valores logarítmicos, afim de minimizar a variabilidade do desvio de uma observação comparativa quanto aos resultados dos parâmetros microbiológicos. Em seguida, foi realizado o pressuposto de normalidade dos dados, a partir do teste de ShapiroWilk. Observa-se na Figura 2, que os parâmetros microbiológicos das 31 amostras agrupadas da FMF não seguem distribuição normal, logo, os parâmetros de coliforme total e bolores termorresistentes apresentaram a menor e maior variabilidade respectivamente, e com valor médio $\left(\mathrm{x}^{-}=0.88 \mathrm{UFC} / \mathrm{g}\right)$, próximo do valor de referência mediano $(\tilde{\mathrm{x}}=0.87 \mathrm{UFC} / \mathrm{g})$ para o coliforme total, entretanto, sugere-se que distribuição é assimétrica negativa (A_S = -0.147).

Para a detecção de Salmonella spp., todas as 31 amostras pesquisadas apresentaram ausência desse enteropatógeno em 25 gramas de FMF. Se nos ensaios biológicos for detectado a presença do gênero Salmonella, as amostras devem ser descartadas de forma segura pelas empresas produtoras, haja visto que a presença deste patógeno, representa grave risco sanitário. De acordo com Santos et al. (2020) no Brasil, o gênero Salmonella é um dos principais agentes causador de doença envolvida nos surtos de DTAs, e a espécie entérica desse gênero é a que mais acomete os seres humanos, através do consumo de água e alimentos contaminados. Portanto, a prevenção de surtos de salmonelose é de grande relevância na saúde pública, pois nem todos os surtos que ocorrem, há notificação nos informes oficiais.

$\mathrm{Na}$ investigação de Escherichia coli e Bacillus cereus, também não foram isolados essas bactérias em nenhuma das amostras analisadas. Caso houvesse sido detectada a presença desses enteropatógenos, estes poderiam representar risco biológico capaz de provocar graves surtos de gastroenterite alimentar (Forsythe, 2013; Jay, 2005; Franco, Landgraf, 2008). De acordo com a Anvisa (2019), os agentes etiológicos mais identificados nos surtos de DTA no Brasil de 2009 a 2018, foram a $E$. Coli e B. cereus, responsáveis por 23,4\% e 2,3\% respectivamente, dos surtos investigados pela Vigilância Sanitária no Brasil. 
Figura 2: Box plot da variabilidade estatística em escala logarítmica dos parâmetros microbiológicos em 31 amostras agrupadas de farinha flocada de milho. Fonte: Própria, 2020.

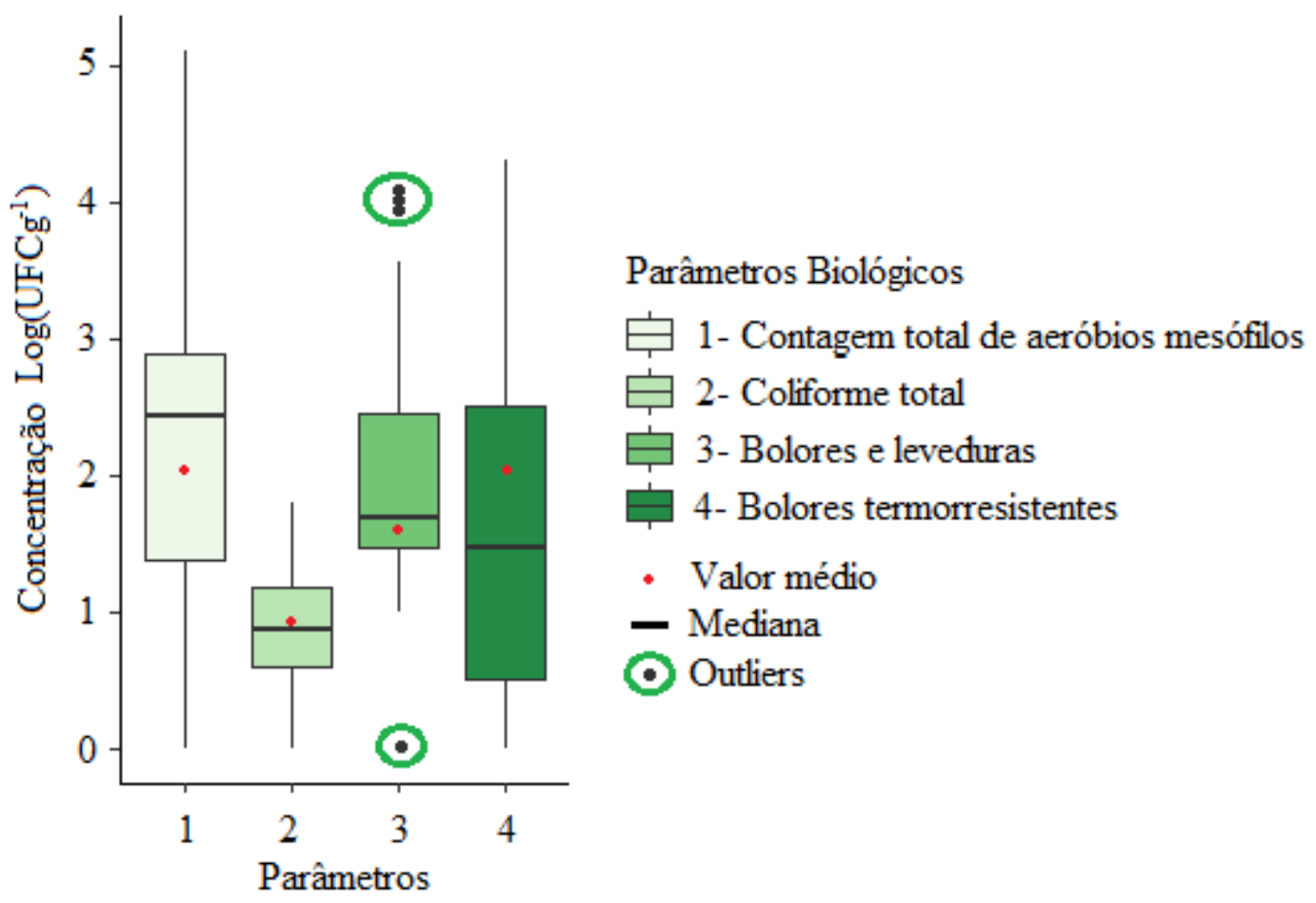

Fonte: Autores.

Quanto a pesquisa de contagem padrão de aeróbios mesófilos e coliforme total (FIGURA 2), foram detectadas concentrações inferiores a log 3 UFC.g-1, que segundo Franco (2012) e Tondo e Bartz (2012), esses valores encontrados atendem parâmetros biológicos considerados seguros, para atividade de manipulação e produção de alimentos para o consumo humano.

Quanto a Contagem Padrão de Aeróbios Mesófilos em um produto alimentício, a presença destes refletem a qualidade da matéria-prima, bem como expressa as condições de processamento, manuseio e estocagem, permitindo estimar o tempo de prateleira do alimento em questão, e também as características sanitárias do produto final. Esse grupo é composto por agentes biológicos deteriorantes, afetando diretamente a vida útil do alimento (Massaguer, 2005; Sant'ana, Conceição, Azeredo, 2002; Silva et al., 2017).

A pesquisa de coliforme total nos alimentos informa a qualidade higiênico-sanitária do ambiente fabril e a presença da E. Coli indica contaminação por material fecal, ambos representam parâmetros sanitários importantes a serem avaliados para a determinação da qualidade e comprovação de alimento seguro. Essas informações são úteis para identificar e mapear se as boas práticas de fabricação estão implementadas, bem como para detectar alguma possível presença de enteropatógenos, que pode estar associada com a presença de microbiota entérica oriunda de contaminação cruzada (Bergaglio, Bergaglio, 2020; Kim et al., 2017). As condições higiênicas do ambiente de trabalho e o cumprimento das exigências oficiais, são fatores importantes na produção e comercialização de alimentos seguros e de qualidade comprovada.

Observa-se na Figura 2, que a contagem de bolores e leveduras apresenta resposta que se diferenciam de todos os outros ensaios microbiológicos, onde 3 (três) Outliers se destacaram dos demais parâmetros, deve-se ressaltar que mesmo sendo esses dados discrepantes ou pontos fora da curva normal, ainda apresenta concentração fúngica permitido por norma sanitária, que determina concentração máxima de até $10^{4}$ UFC/grama para diferentes grupos de alimentos (BRASIL, 2019b). Os bolores e leveduras em altas contagens indicam falha no processo de sanitização e processamento dos alimentos ou uma 
seleção inadequada da matéria-prima, introduzindo no processo industrial insumos contaminados por agentes patogênicos (Franco, 2012; Massaguer, 2005; Shinohara et al., 2017).

No caso dos bolores e leveduras, geralmente apresentam baixa resistência ao calor, sendo facilmente destruídos frente aos tratamentos térmicos empregados na indústria de alimentos (Shinohara et al., 2017). Entretanto, há certos fungos que produzem ascósporos, estrutura de resistência capaz de sobreviver a tratamentos térmicos, congelamento convencionais e pressão osmótica (Massaguer, 2005; Tortora, Funke, Case, 2016). Alguns desses fungos filamentosos termófilos produzem as micotoxinas, metabólitos secundários, que apresentam atividade mutagênica, carcinogênica e teratogênica, sintetizados no final da fase de crescimento exponencial (Iamanaka, Oliveira, Taniwaki, 2010).

Quanto às toxinas fúngicas, a $\mathrm{RDC} \mathrm{n}^{\circ} 7$ (Brasil, 2011) recomenda que os níveis de micotoxinas deverão ser o mais baixos quanto razoavelmente possível, devendo ser aplicadas as melhores práticas e tecnologias na produção, manipulação, armazenamento, processamento e embalagem, de forma a evitar que um alimento contaminado por esses metabólitos secundários, estejam em níveis superiores aos recomendado por essa resolução, ou seja, que os alimentos em concentrações acima de limites seguros, não sejam comercializado e nem consumido pela população brasileira.

Na Etiópia, país do continente africano, foram isolados espécies de fungos no milho estocado, armazenados sem a adoção das boas práticas agrícolas, de transporte e armazenamento adequados, propiciando assim o crescimento de fungos filamentosos, Aspergillus spp. e Fusarium spp., decorrente do aumento da umidade relativa durante o armazenamento. Esses gêneros fúngicos são capazes de produzir micotoxinas e comprometer a segurança alimentar dos consumidores (Negasa, Solomon, Girma, 2019; Silva Jr, 2014).

No Brasil, dentre os fungos mais encontrados no milho e derivados, pertencem ao gênero Fusarium e suas espécies, microrganismos com potencial produção da micotoxina fumonisinas, composto químico com elevado potencial e capacidade hepatotóxica e carcinogênicas e ainda pouco investigada na saúde coletiva dos países produtores de cereais. O conhecimento dos níveis de contaminação por fumonisinas no milho se torna muito importante para tomada de decisão, levando em consideração a qualidade do milho disponível no mercado. Ressalta-se a necessidade de inserção de métodos alternativos no monitoramento de micotoxinas, e desta forma facilitar o controle nas indústrias de processamento (Oliveira et al., 2020).

Em relação aos grupos vulneráveis, existem evidências científicas que a população idosa, por causa do avanço da idade, fica mais susceptível às infecções veiculadas por água e alimentos, devido ao declínio da imunidade e das funções fisiológicas (Leite, Waissmann, 2006). As doenças transmitidas por alimentos (DTA), são subnotificadas no Brasil, a Organização Mundial da Saúde (OMS) estima que 1/3 da população adoeça anualmente de DTA, entretanto, apenas uma pequena parcela desses surtos são notificados em informes oficial (Viterbo et al., 2020). Com o aumento da ocorrência das doenças transmitidas por alimentos e água, cenário que evolui para graves consequências aos grupos populacionais mais expostos, gerando repercussões econômicas, como despesas médicas, ausência ao trabalho e quebra de produtividade, o que representa um problema de saúde pública (Santos et al., 2017).

\section{Conclusão}

Os ensaios biológicos realizados na farinha de milho flocada na RGR, informam que na pesquisa de patógenos obrigatórios, para garantia de produção e comercialização segura de alimentos, as amostras estão em conformidade com os parâmetros sanitários previstos em legislação no Brasil. Entretanto, outros microrganismos patogênicos ou deteriorantes podem estar presentes nesse grupo de alimento, o que caracteriza risco biológico, principalmente para grupos de pessoas que apresentam vulnerabilidade e susceptibilidade, frente às contaminações biológicas dos alimentos. 


\section{Referências}

Amaral, L. F., \& Ferreira, I. M., \& Santos, N., L. V., \& Oliveira, A. M., \& Fagundes, A. A., \& Carvalho, M. G. (2019) Biscoito com especiarias e farinhas de milho e semente de abóbora: desenvolvimento e avaliação da qualidade. DEMETRA: Alimentação, Nutrição \& Saúde, $14,33380.1$-17.

ANVISA. Agência Nacional de Vigilância Sanitária. <https://portalarquivos2.saude.gov.br/images/pdf/2019/fevereiro/15/Apresenta----o-Surtos-DTA--Fevereiro-2019.pdf>.

APHA. (2005) American Public Health Association. Standard methods for the examination of water and wastewater. (21st ed). Washington, DC.

Araújo, W. M. C. \& Montebello, N. P. \& Botelho, R. B. A. \& Borgo, L. A. (2016) Alquimia dos Alimentos. Senac.

Ayeni, K. I., Akinyemi, O. M., Kovač, T., Ezekiel, C. N. (2020) Aflatoxin contamination of maize vended in Ondo state, Nigeria, and health risk assessment. Croatian journal of food science and technology, 12, 123-129.

Bergaglio, J., \& Bergaglio, O. (2020) Contaminación de alimentos por Escherichia coli y la inocuidad alimentaria como eje fundamental. INNOVA UNTREF, (5), 2-17.

Brasil. Agência Nacional de Vigilância Sanitária. Resolução da Diretoria Colegiada - RDC N ${ }^{\circ} 150$ (2017). Dispõe sobre o enriquecimento das farinhas de trigo e de milho com ferro e ácido fólico. < https://www.in.gov.br/materia/asset_publisher/Kujrw0TZC2Mb/content/id/20165624/do1-2017-04-17-resolucaordc-n-150-de-13-de-abril-de-2017-20165414>.

Brasil. Resolução $\mathrm{N}^{\mathrm{O}} 7$ (2011). Dispõe sobre limites máximos tolerados (LMT) para micotoxinas em alimentos. <http://bvsms.saude.gov.br/bvs/saudelegis/anvisa/2011/res0007_18_02_2011_rep.html>.

Brasila. Resolução da Diretoria Colegiada - RDC No 331 (2019). Dispõe sobre os padrões microbiológicos de alimentos e sua aplicação. http://portal.anvisa.gov.br/documents/10181/4660474/RDC_331_2019_COMP.pdf/c9282210-371f-4fb6-b343-7622ca9ec493>.

Brasilb. Instrução Normativa - N 60 (2019). Estabelece as listas de padrões microbiológicos para alimentos. <http://www.in.gov.br/en/web/dou/-/instrucaonormativa-n-60-de-23-de-dezembro-de-2019-235332356>.

Cascudo, L. C. (1988) Dicionário do Folclore Brasileiro. Edusp.

CONAB. Companhia Nacional de Abastecimento. Acompanhamento da Safra Brasileira de Grãos (2020). V7 Safra 2019/2020. <file:///C:/Users/Microsoft/Downloads/GrosZabrilZresumoZ2020-4.pdf>.

Forsythe, S. J. (2013). Microbiologia da Segurança dos Alimentos. Artmed.

Franco, B. D. G. M.,\& Landgraf, M. (2008) Microbiologia dos Alimentos. Atheneu.

Franco, R. M. (2012) Agentes Etiológicos de Doenças Alimentares. Editora UFF.

Freixa, D., \& Chaves, G. (2012) Gastronomia no Brasil e no Mundo. Senac.

Germano, P. M., \& Germano, I. S.(2019) Higiene e Vigilância Sanitária de Alimentos. Manole.

Iamanaka, B. T. \& Oliveira, I. S., \& Taniwaki, M. H. (2010) Micotoxinas em alimentos. Anais da Academia Pernambucana de Ciência Agronômica. v7, p.138-161.

ICMSF. (2002) International Commission On Microbiological Specifications for Foods. Microrganisms in Food 7. Micobiological Testing in Food Safety Management. Kluwer Academic/ Plenum Publishers, (ISBN: 0-306-47262-7).

Jay, J. M.(2005) Microbiologia de Alimentos. Artmed.

Kim, H. W., Hong, Y. J., Jo, J. I., Ha, S. D., Kim, S. H., Lee, H. J., Rhee, M. S. (2017) Raw ready-to-eat seafood safety: microbiological quality of the various seafood species available in fishery, hyper and online markets. Letters in applied microbiology, 64(1), 27-34.

Kovesi, B., Siffert, C., Crema, C., Martinoli, G. (2007) 400g: técnicas de cozinha. Editora Nacional.

Leite, L. H. M.; Waissmann, W. (2012) Doenças transmitidas por alimentos na população idosa: riscos e prevenção. Revista de Ciências Médicas, 15, 525530 .

Massaguer, P. R. (2005) Microbiologia dos Processos Alimentares. Livraria Varela.

Negasa, F., Solomon, A., Girma, D. (2019) Effect of traditional and hermetic bag storage structures on fungus contamination of stored maize Grain (Zea mays L.) in Bako, Western Shoa, Ethiopia. African Journal of Food Science, 13(3), 57-64.

Oliveira, D. H. et al. (2020) Ocorrência de fumonisinas em milho e derivados, destinados à alimentação humana/Ocurrence of fumonisin in corn and derivates, intended for human consumption. Brazilian Journal of Development, 6, 31305-31321.

Onyedum, S. C. et al. (2020). Occurrence of major mycotoxins and their dietary exposure in North-Central Nigeria staples. Scientific African, 7 , e00188. <https://doi.org/10.1016/j.sciaf.2019.e00188>.

Sant'ana, A. S., Conceição, C., Azeredo, D. R. P. (2002) Comparação entre os métodos rápidos SimplateR TPC- CI e PetrifilmR AC e os métodos convencionais de contagem em placas para a enumeração de aeróbios mesófilos em sorvetes. Food Science and Technology, $22,60-64$. 
Research, Society and Development, v. 10, n. 2, e31010212585, 2021

(CC BY 4.0) | ISSN 2525-3409 | DOI: http://dx.doi.org/10.33448/rsd-v10i2.12585

Santos, J. M. R., Ribeiro, M. C., Ribeiro, G. C, Souza, A. P. F., do Nascimento, C. D., Lopes, R. C. R. (2017) Estudo sobre a ocorrência de surtos alimentares em uma região do Vale do Jequitinhonha, Minas Gerais. Vigilância Sanitária em Debate: Sociedade, Ciência \& Tecnologia, 5, 30-36.

Santos, K. P. O., Faria, A. C. D. S. R., Silva, D. P. A., Lisboa, P. F., Costa, A. P, Knackfuss, F. B. (2020) Salmonella spp. como agente causal em Doenças Transmitidas por Alimentos e sua importância na saúde pública: Revisão. PUBVET, 14(10), 1-9.

Santos, M. C. L., Furtado, A. F. T. L., Shinohara, N. K. S. (2019) Avaliação da rotulagem de flocos de milho pré-cozidos do tipo "Flocão". Journal of Environmental Analysis and Progress, 4, 257-265.

Santos, M. C. L., Mendonça, C. M., Shinohara, N. K. S. (2020) Milho e São João: Identidade Gastronômica. Contexto da Alimentação, Cultura e Sociedade, $8(1)$.

Shapiro, S. S., \& Wilk, M. B. (1965) An analysis of variance test for normality (complete samples). Biometrika, 52(3/4), 591-611.

Silva Júnior, E. A. (2014) Manual de Controle Higiênico-sanitário em Serviços de Alimentação. Varela.

Silva, N., Junqueira, V., Silveira, N., Taniwaki, M., Gomes, R., Okazaki, M. (2017) Manual de Métodos de Análise Microbiológica de Alimentos e Água. (5a ed.) Blucher.

Simões, G., Demérito, G. B., Paula, G. F., Ladeira, D. C., Matsumoto, L. S. (2020) Influence of spent coffee grounds on soil microbiological attributes and maize crop. Research, Society and Development, 9(8), e818986400-e818986400.

Tiecker, A., Guimarães, L. E., Ferrari Filho, E. D. A. R., Castro, B., Del Ponte, E. M. E. R. S. O. N Dionello, R. et al. (2014) Qualidade físico-química de grãos de milho armazenados com diferentes umidades em ambiente hermético e não hermético. Revista Brasileira de Milho e Sorgo, 13, 174-186.

Tondo, E. C., \& Bartz, S. (2012) Microbiologia e Sistema de Gestão da Segurança de Alimentos. Sulina.

Tortora, G. J., Funke, B. R., CASE, C. L. (2016) Microbiologia. Artmed.

Ventura, M. V. A., Batista, H. R. F., Bessa, M. M., Costa, E. M., Pereira, L. S., \& Silva, R. M. (2020) Production cost of transgenic corn in the first and second harvest in different regions in Brazil. Research, Society and Development, 9(7), e279974136-e279974136.

Viterbo, L. M. F., Dinis, M. A. P., Sá, K. N., Marques, C. A. S. D. C., Navarro, M. V. T., \& Leite, H. J. D. (2020) Desenvolvimento de um instrumento quantitativo para inspeção sanitária em serviços de alimentação e nutrição, Brasil. Ciência \& Saúde Coletiva, 25, 805-816.

Ximenes, G. N. C., Cortes, N. M. S., Shinohara, G. M., Padilha, M. R. F. (2017) Pesquisa de Fungos em Farinha de Trigo Comercializada na Região Metropolitana do Recife-PE. Nutrição em Pauta, 7(1), 43-56. 